\title{
Enhancing elderly mobility through IoT using textiles: a review
}

\author{
M. J. Magalhães ${ }^{1,2}$, Sérgio Tenreiro de Magalhães ${ }^{3,4,5}$ and Kenneth Revett ${ }^{6}$ \\ ${ }^{1} 2 \mathrm{C} 2 \mathrm{~T}$ - University of Minho, Guimarães, Portugal, mariajmrm@gmail.com \\ ${ }^{2}$ Faculdade de Filosofia e Ciências Sociais da Universidade Católica Portuguesa, Braga, \\ Portugal, mjmagalhaes@braga.ucp.pt \\ ${ }^{3}$ ALGORITMI Reseach Center - University of Minho, Guimarães, Portugal, \\ psmagalhaes@dsi.uminho.pt \\ ${ }^{4}$ Faculdade de Filosofia e Ciências Sociais da Universidade Católica Portuguesa, Braga, \\ Portugal, stmagalhaes@braga.ucp.pt \\ ${ }^{4}$ Department of Computer Science, University of Beira Interior, Covilhã, Portugal, \\ stmagalhaes@di.ubi.pt \\ ${ }^{6}$ Anastamose International, Boston, MA USA, Ken.revett@gmail.com
}

\begin{abstract}
The aging of population worldwide and the increasing of average life expectancy of the world population become a social and economic problem for society. It's necessary to provide solutions which can maintain the independency of elderly and their mobility, along more time, avoiding the unnecessary permanence in hospitals and institutions for the elderly. The use of IoT using textiles is a very interesting approach because of the proximity of people to these materials. This work reviews the development on IoT using textiles to increase the mobility of elderly people and concludes that this is a field of growing interest. Although, there is few investigation, especially in what concerns studies focusing on the importance of improving the mobility of the elderly. There are studies that lead to the possibility of promoting this physical capability but have not been designed for that purpose, but we believe that approach of the problem of mobility of elderly people using the IoT based on clothes should be treated with specificity, because the consequences of this not happening, will affect not only the elderly but the whole society.
\end{abstract}

Keywords: Internet of Things (IoT), Smart Clothing, Smart Textiles, Mobility, Elderly 


\section{Introduction}

The average life expectancy of the world population has increased [1] generating an increase in social concerns with the elderly. The increasing number of elderly people generates the need to create various supporting infrastructure in order to support this age group. Older people have specific characteristics, such as the increase of diseases, reduced mobility, physical changings, etc. [2]. All these features reduce the independence of the elderly that see their mobility reduced by fears of falling, physiological changes associated with some chronic disease, fear of being lost due to loss of cognitive abilities, etc. The IoT (Internet of Things) presents a number of opportunities that allow to support the elderly in their everyday life increasing their mobility and, in consequence, increasing their quality of life. The quality of life of the elderly is related to their health, to their physical limitations, but also with the loneliness factor [3]. In his study, Farquhar concluded that elderly people consider de social contact so important as health on quality of life [4]. In the same study one of the very elderly respondents said: "I miss someone to be able to talk to, to make decisions with ... I don't like to be so lonely" [4]. The reduction of their mobility will make them live increasingly in solitude and this is one of the most important problems of older people [5] [6]. The use of IoT can allow the elderly to move without fear, because different information about them can be monitored remotely by caregivers, family members or doctors.

The IoT use tiny sensors connected to internet allowing the transmission of information. The sensor converts the mechanical measure into an electrical output. The use of elements that are applied directly in the body of the elderly for capturing/communicating of data, such as sensors, may bring problems of skin sensitivity, because of the characteristics of the skin of the elderly, which is more sensitive and more susceptible to allergies. Thus, the use of clothing, which is part of everyday life of persons, appears to be the most natural form to integrate de electronic devices. For example, the use of a bra that has an embedded control device and transmits the information on the rate or cardiac changes, has no inconvenience to the elderly women and is part of their daily dress [7].

Smart textiles are textiles that feel and respond to the changes that occurs in their environment. There two types of smart textiles: passive and active. The active smart textiles are fitted with sensors and actuators to receive the change and transmit the information. The market of IoT expect about 30 billion of devices connected in 2020 , above $10 \%$ of those will be clothes. That 3 billion clothes connected will use smart textiles, improving the industry [8].

In order to study the research work that has been published regarding the use of IoT using clothing for elderly with the objective of making possible to promote their mobility, a literature review was conducted. The next section presents the method used to conduct that review. Section 3 presents the results obtained on the level of research in the topic and the analysis of the contents of the existing re- 
search. Finally, conclusions are taken from the research currently available in peer-reviewed indexed publications (Scopus and/or ISI Web of Science).

\section{Method}

For the literature review several queries were made during the months of August and September 2016, of publications indexed in SCOPUS and in the ISI Web of Science.

On SCOPUS, various keywords and various operators were used. All "subject areas" were considered.

The different combination of keywords was made using the field "abstracts, keywords and titles", in different phases which will be described below:

- Phase I - The authors made all the possible combinations between the Fields 1, 2 and 3 defined below, using the operator AND:

Field 1 - Internet of Things

Field 2 - Elderly / Elder / Older

Field 3 - Clothes / Clothing / Garment / Textiles

The result included 3 documents (Table 1).

Table 1. - Results from Phase I

\begin{tabular}{lc}
\hline \multicolumn{1}{c}{ Terms used in the query } & Number of documents found \\
\hline Internet of Things + Elderly + Clothes & 1 \\
Internet of Things + Older + Clothing & 1 \\
Internet of Things + Older + Textiles & 1 \\
\hline
\end{tabular}

- Phase II - The authors made all the possible combinations between the Fields 1, 2 and 3 defined below, using the operator AND:

Field 1 - Internet of Things

Field 2 - Elderly / Elder / Older

Field 3 - Smart Clothes / Smart Clothing / Smart Textiles

The result included 2 documents (Internet of Things + Older + Smart Textiles).

- Phase III - The authors made all the possible combinations between the Fields 1,2 and 3 defined below, using the operator AND:

Field 1 - Internet of Things

Field 2 - Elderly / Elder / Older

Field 3 - Technical Clothes / Technical Clothing / Technical Textiles 
The result included 1 document (Internet of Things + Older + Technical Textiles).

The Phases I, II and III were repeated replacing the expression "Internet of Things" for IoT. The number of documents found was zero.

At this point the authors decided to give up on words related with elderly people and look for more general combinations.

- Phase IV - The authors made all the possible combinations between the Fields 1 and 2 defined below, using the operator AND:

Field 1 - Internet of Things

Field 2 - Smart Clothes / Smart Clothing / Smart Textiles

The result included 35 documents (Table 2).

Table 2. Results from Phase IV

\begin{tabular}{lc}
\hline \multicolumn{1}{c}{ Terms used in the query } & Number of documents found \\
\hline Internet of Things + Smart Textiles & 13 \\
Internet of Things + Smart Clothes & 9 \\
Internet of Things + Smart Clothing & 13 \\
\hline
\end{tabular}

- Phase V - The authors made all the possible combinations between the Fields 1 and 2 defined below, using the operator AND:

Field 1 - Internet of Things

Field 2 - Technical Clothes / Technical Clothing / Technical Textiles

The result included 7 documents (Table 3).

Table 3. Results from Phase V

\begin{tabular}{lc}
\hline \multicolumn{1}{c}{ Terms used in the query } & Number of documents found \\
\hline Internet of Things + Technical Textiles & 5 \\
Internet of Things + Technical Clothes & 1 \\
Internet of Things + Technical Clothing & 1 \\
\hline
\end{tabular}

The Phases IV and V were repeated replacing the expression "Internet of Things" for IoT and 13 documents were found with the new combination for the replacement on Phase IV - Phase VI (Table 4) - and 3 documents for the replacement on Phase V - Phase VII (Table 5). 
Table 4. Results from Phase VI

\begin{tabular}{lc}
\hline Terms used in the query & Number of documents found \\
\hline IoT + Smart Textiles & 5 \\
IoT + Smart Clothes & 2 \\
IoT + Smart Clothing & 6 \\
\hline
\end{tabular}

Table 5. Results from new Phase VII

\begin{tabular}{lc}
\multicolumn{1}{c}{ Terms used in the query } & Number of documents found \\
\hline IoT + Technical Textiles & 1 \\
IoT + Technical Clothing & 2 \\
\hline
\end{tabular}

In all the Phases the authors tried to insert a new field with the keyword "mobility" but the results were always zero documents.

Finally, it was used a tool from the SCOPUS platform that makes a logical disjunction between the lists of documents obtained on the seven Phases (I, II, III, IV, $\mathrm{V}, \mathrm{VI}$ and VII) of the queries to exclude the repeated documents. The result was 35 documents that were filtered by "Document Type". The authors opted to analyse "Article", "Conference paper" and "Book Chapter" types. The final list of documents had 26 documents.

The next step of the work consisted in reading the abstracts of all the 26 final documents, or the total document when it was needed, to exclude the ones which were out of the scope of this work. After analyzing all 26 documents, 7 remained as the final documents.

The same Phases of queries were made in ISI Web of Science, but the documents found either were out of the scope or they were already included in the ones obtained on the SCOPUS queries. 


\section{Results}

\subsection{The evolution of research on the topic}

The first year that has published documents about the topic was 2010 and since 2014 the number of documents has been growing. $71.4 \%$ of the documents were published in the last 2 years and 57.1\% in 2016 (until June), which shows an increasing interest in the topic (Figure 1).

Fig. 1. Evolution of the publication of documents since 2010

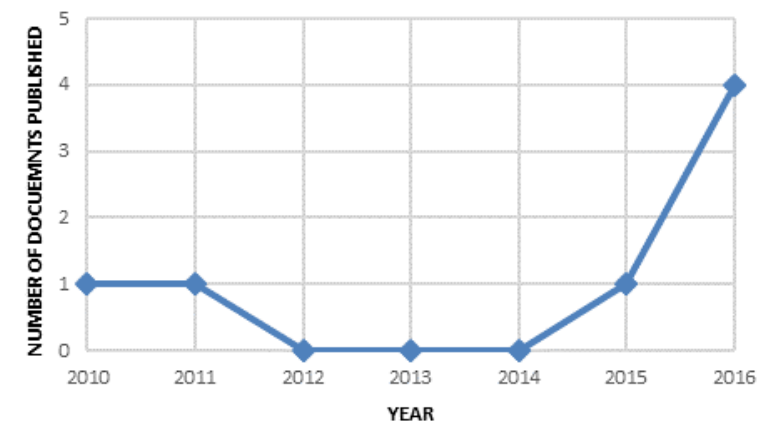

The topic has captured the interest of researchers from various countries, with researchers from six countries and three continents (America, Asia and Europe). China is the country with more documents (Figure 2). The number of documents analysed and the number of documents presented in Figure 2 are different because some documents have more than one author, and they can be from different countries.

Fig. 2. Documents published by the countries 


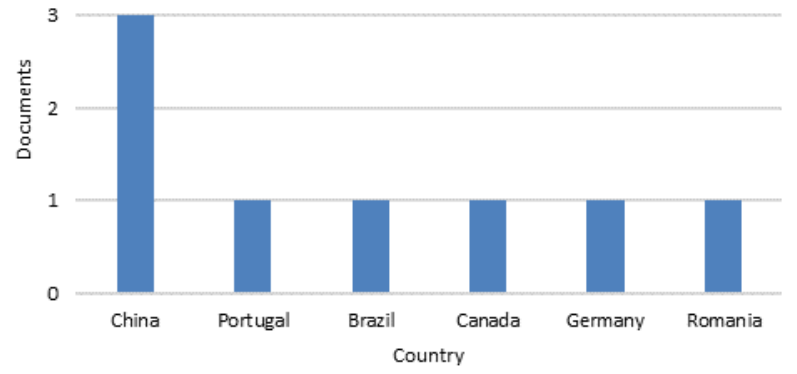

Some documents are classified in more than one subject area; this is the reason why the number of documents is higher in the Figure 3 than the ones which were analysed. There are six scientific domains and the more representatives (42.9\%) are the Computer Science, Engineering and Materials Science.

Fig. 3. Documents published by the subject area

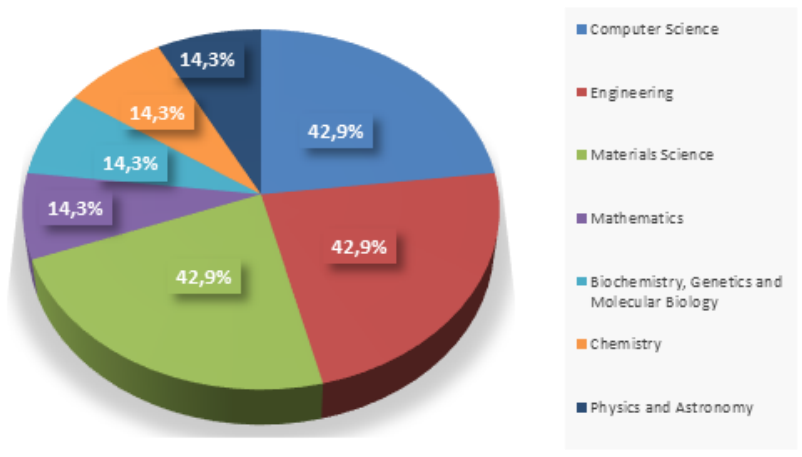

\subsection{Results from the analyses of the documents}

When we are talking about smart clothes, the necessity of energy harvesting is very important because of the difficulty of using and replacing a battery. Loss et al presented a smart and sustainable coat that has a dual-band textile antenna embedded in the textile, that operates at global system for mobile communication (GSM) 900 and digital cellular system (DCS) 1800 bands. The antenna is printed 
in the textile material by embroidering or lamination. This study shows that the textile works like a dielectric and doesn't affect the performance of the antenna. The lamination is preferable to embroidering because it's more plane, although, in the case of embroidering, the number and directions of the stitches can influence the signal, increasing the conductivity of some elements. The integration of the electronic device for communication in the garments by the printing in the clothes, its more discreet and comfortable for the user. The evolution of antennas making them smaller, cheaper and flexible is very important because it permits that they can be incorporated in clothing [9]

To integrate sensors in smart clothes they have to be small, the antenna has to be small and flexible, the capacity of alimentation of the device must be guaranteed but small at the same time, the distance between the sensors and the body and between sensors and the sink nodes must be short, and there are questions of information security [10].

For WBSN (Wireless Body Sensor Networks) applications there are flexible textile biosensors: woven/knitted metal electrodes (surface electrode as sensor), electroactive polymeric fibre (piezoresistive sensor as sensor), PVDF film or woven patch (piezoelectric microphone as sensor), etc. Biosensors permit to measure the respiration, blood oxygenation, skin temperatures, skin electrical impedance, heart sound, etc. [10]

Chen used smart clothing in a study to collect the small electrical changes that occurs in the skin of humans in consequence of the activity of the human heart electrocardiogram (ECG). Those measures permit to evaluate the users' emotions. The objective of use smart clothing, where the sensors are integrated to collect the body signals, is to maximize the comfort of the users. In this research project, the authors designed a CP-Robot system that allows, using smart clothing, smart phones and assisted by the cloud, that two users can connect with the real perception of the mood/emotions of the other person [11].

Older people want to be independent, live in their homes with their family and not in an institution [12]. The enormous development on ICT (Information and Communications Technology) will help to maintain the older people independent and with mobility for more time. The use of sensors and IoT to support living at home changed the concept of Ambient Assisted Living (AAL). Although, what seems to be a response to the problem of older people living alone, can be a problem too. The existence of "to much" technology inside the homes, especially when we are talking about elderly, may not be accepted by everyone. Ziefle tried to realize if the introduction of smart textiles input devices will be accepted on an AAL. The study was made using a large range of ages to evaluate if there are differences between younger and older people. Results shows that smart textiles were considered less enjoyable in the kitchen and in the bedroom and more in the living room. The use of smart textiles integrated in clothes was more enjoyable too. About the influence of the age, no relation was found between age and acceptance of this technology [13].

Zheng defines e-textiles as smart fabrics woven that can incorporate electronic devices, inter-connectors and power supplies, which are materials base of IoT. The 
textile industry has capability to produce those smart fabrics but it must understand all the components integrated on the e-textiles in order not to damage the electronic compounds. This is a challenge to traditional textiles industries but the differentiation of products is also the future of textiles [14].

\section{Conclusions}

The human being is accustomed to the contact with textiles in his day-to-day: clothes, shoes, fashion accessories, home textiles, the interior of the cars, etc. The use of electronic devices integrated in the clothes, in a comfortable way, seems a very natural choice. The acceptance of using e-textiles at home integrated in a AAL had a good result, but with different acceptance depending on the space of the house. Also the studies didn't find any relation between age and acceptance, what means that the acceptance by elderly to the use of IoT using smart textiles is expected be positive.

Studies also shows that there is a panoply of smart clothing prepared to support older people to maintain themselves independent and with mobility to go out of their homes, because if something goes wrong the device will alert a doctor, a relative or the caregiver about the situation and with a GPS integrated on the smart clothing it will be easy to locate the person.

The study of Chen was also very interesting because it presents a novelty that can be a way to overcome loneliness with a better approximation of distance relation with real emotions. Most of the times the transmission of emotion gets lost in the process.

The use of IoT to support elderly using the smart clothes permits them to be happier, with a better quality of life, involving independency and capability of moving by themselves. Increase the mobility of elderly is at the same time a contribution to the decrease of loneliness, one of the biggest problems of elder people nowadays, a contribution to a better health (physical and psychological) and a contribution to the society. The research on this theme is starting, but the aging population worldwide will precipitate the industry and institutions to adhere and develop quicker IoT using clothing for this propose.

Acknowledgments This work has been supported by COMPETE: POCI-01-0145-FEDER007043 and FCT - Fundação para a Ciência e Tecnologia within the Project Scope: $\mathrm{UID} / \mathrm{CEC} / 00319 / 2013$

\section{References}

[1] Commission, E. (2016). Eurostat. Retrieved September 27, 2016, from http://appsso.eurostat.ec.europa.eu/nui/show.do?dataset=demo_mlexpec\&lang=en 
[2] WALKER, A. (Ed.). (2014). The new science of ageing, (Bristol: Policy Press).

[3] TAUBE, E., JAKOBSSON, U., MIDLÖV, P., \& KRISTENSSON, J. (2016). Being in a Bubble: the experience of loneliness among frail older people. Journal of Advanced Nursing 72(3): 631-640.

[4] FARQUHAR, M. (1995) Elderly people's definitions of quality of life. Social science \& medicine 41(10): 1439-1446.

[5] TIANG, Q. (2016). Intergeneration social support affects the subjective well-being of the elderly: Mediator roles of self-esteem and loneliness. Journal of Health Psychology 21(6): $1137-1144$.

[6] GABRIEL, Z., \& BOWLING, A. (2004). Quality of life from the perspectives of older people. Ageing and Society 24(05): 675-691.

[7] (2008). New uses for wearable textile-based health monitoring technology. (2008). Textile Outlook International 135, 87-88.

[8] KONCAR, V. (2016). Introdution to smart textiles and their applications, In KONCAR, V. (Ed.), Smart Textiles and Their Applications (Duxford: WoodHead Publishing), ch.1.

[9] LOSS, C., GONÇALVES, R., LOPES, C., PINHO, P., \& SALVADO, R. (2016). Smart Coat with a Fully-Embedded Textile Antenna for IoT Applications. Sensors 16(6), 938

[10] KIM, Y. K., WANG, H., \& S., M. (2016). Wearable body sensor network for health care applications, In KONCAR, V. (Ed.), Smart Textiles and Their Applications (Duxford: WoodHead Publishing), ch.9.

[11] CHEN, M., MA, Y., HAO, Y., LI, Y., WU, D., ZHANG, Y., \& SONG, E. (2016) Cprobot: Cloud-assisted pillow robot for emotion sensing and interaction. In International Conference on Industrial IoT Technologies and Applications March 2016 (Springer International Publishing), 81-93

[12] DEWSBURY, G., \& EDGE, M. (2001) Designing the Home to Meet the Needs of Tomorrow. Open House International 26(2): 33-42.

[13] ZIEFLE, M., BRAUNER, P., \& VAN HEEK, J. (2016) Intentions to Use Smart Textiles in AAL Home Environments: Comparing Younger and Older Adults. In International Conference on Human Aspects of IT for the Aged Population July 2016 (Springer International Publishing), 266-276.

[14] ZHENG, N. G. (2011). A survey on electronic textiles. Jisuanji Xuebao (Chinese Journal of Computers) 34(7): 1172-1187. 